\title{
A Novel Learning Based Model Predictive Control Strategy for Plug-in Hybrid Electric Vehicle
}

\author{
Yuanjian Zhang, Member, IEEE, Yanjun Huang, Member, IEEE, Zheng Chen, Senior Member, IEEE \\ Guang Li, Member, IEEE, and Yonggang Liu, Senior Member, IEEE
}

\begin{abstract}
The multi-source electromechanical coupling renders energy management of plug-in hybrid electric vehicles (PHEVs) highly nonlinear and complex. Furthermore, the complicated nonlinear management process highly depends on knowledge of driving conditions, and hinders the control strategies efficiently applied instantaneously, leading to massive challenges in energy saving improvement of PHEVs. To address these issues, a novel learning based model predictive control (LMPC) strategy is developed for a serial-parallel PHEV with the reinforced optimal control effect in real time application. Rather than employing the velocity-prediction based MPC methods favored in the literature, an original reference-tracking based MPC solution is proposed with strong instant application capacity. To guarantee the optimal control effect, an online learning process is implemented in MPC via the Gaussian process (GP) model to address the uncertainties during state estimation. The tracking reference in LMPC based control problem in PHEV is achieved by a microscopic traffic flow analysis (MTFA) method. The simulation results validate that the proposed method can optimally manage energy flow within vehicle power sources in real time, highlighting its anticipated preferable performance.

Index Terms - Optimal control strategy, leaning based model predictive control (LMPC), Gaussian process (GP) model, microscopic traffic flow analysis (MTFA), plug-in hybrid electric vehicle (PHEV).
\end{abstract}

\section{INTRODUCTION}

$\mathrm{N}_{\mathrm{s}}$ OWADAYs, plug-in hybrid electric vehicle (PHEVs) have boosted to be top-ranking solutions in auto industry to promote energy consumption economy and mitigate global warming concern $[1,2]$. The tailored powertrain configurations endow PHEVs with extraordinary capacities to leisurely balance driving mileage, drivability, and energy reservation [3]. PHEV, as a complex system with multiple energy degrees of freedom, desperately demands properly designed control strategies to optimally manage energy flow within different sources for maximizing energy saving potentials. The specific

This work was supported in part by the National Key R\&D Program of China (No. 2018YFB0104000), in part by the National Natural Science Foundation of China (No. 61763021 and 51775063), and in part by the EUfunded Marie Skłodowska-Curie Individual Fellowships Project under Grant 845102-HOEMEV-H2020-MSCA-IF-2018. (Corresponding Authors: Zheng Chen, and Yonggang Liu)

Yuanjian Zhang is with the School of Mechanical and Aerospace Engineering, Queen's University of Belfast, BT9 5AG, Northern Ireland. (y.zhang@qub.ac.uk).

Yanjun Huang is with the School of Automotive Studies, Tongji University, Shanghai, 201804, China. (huangyanjun404@gmail.com) electromechanical coupling characteristics in PHEVs, however, embarrass energy management into high nonlinear and complex, laying the knotty challenge waiting to be tackled.

Developing efficient energy management strategies for PHEVs to optimize energy saving is generally a rather difficult task, that needs to account for different constraints from powertrain, driving demand and road conditions. It is intricate to achieve the optimal trade-off among real-time performance, control effect and environmental adaptability. In past years, a variety of efforts have been made to achieve prominent progress in control strategy design of PHEVs. Existing energy management strategies for PHEVs can be mainly divided into the following four categories: rule based control strategies [4, 5], global optimization based strategies [6,7], instantaneous optimization based strategies [8,9] and machine learning based strategies $[10,11]$. For these four types of strategies, despite their validated performance under some specific conditions, it is still intractable to achieve continuous high-quality performance in real-time control, and the potential of PHEVs in energy saving cannot be fully unlocked.

Rule based control strategies, such as threshold methods [4] and fuzzy logic based strategies [5], can be easily constructed and implemented according to the expert knowledge in engineering practice. However, the expert-knowledge based control logics cannot govern powertrain operation in optimal zones all the time. Additionally, the threshold tuning is rather sensitive to driving conditions. Global optimization methods, e.g., dynamic programming (DP) [6] and Pontryagin's minimum principle (PMP) [7], can achieve optimal energy distribution with the known driving conditions. The characterized solving processes globally find the optimal solutions by ergodic search, incurring burdensome computation. On the other hand, the driving knowledge based searching manners make them quite difficult to be applied in real-time problems directly. The instantaneous optimization

Zheng Chen is with the Faculty of Transportation Engineering, Kunming University of Science and Technology, Kunming, 650500, China and also with the School of Engineering and Materials Science, Queen Mary University of London, London E1 4NS, U.K. (e-mail: chen@kust.edu.cn).

Guang $\mathrm{Li}$ is with the School of Engineering and Materials Science, Queen Mary University of London, London, E1 4NS, United Kingdom (e-mail: g.li@qmul.ac.uk).

Yonggang Liu is with the State Key Laboratory of Mechanical Transmissions \& School of Automotive Engineering, Chongqing University, Chongqing, 400044, China (e-mail: andylyg@umich.edu). 
strategies, including equivalent consumption minimization strategy (ECMS) [8] and model predictive control (MPC) [9], are ideal solutions for PHEVs by providing the quasi-optimal effect with the reasonable abilities in real-time implementation. Despite the rational instantaneous control effect, the inner parameters of algorithms and solving mechanisms in these methods entail partial pre-knowledge of future driving conditions. The accuracy of driving conditions identification [8] and prediction [9] in these methods cannot be guaranteed with the limited amount of computation, thus discounting the instantly optimal implementation. Machine leaning based strategies, like Q-learning [10] and Deep Q-learning [11], declare to be optimally implemented without knowing the driving conditions beforehand. The remarkable validation results manifest the massive potential of these methods in PHEV control; however, the offline preparation of these methods costs much effort, and the real application in engineering practice still remains challenging.

The MPC based methods have exhibited strong capabilities in instantaneous applications, and can provide preferable control effect that is rather close to global optimization results. The built-in state estimation models can mitigate the tight dependence on future driving information to a certain extent. MPC has been widely accepted in energy management of PHEVs [12, 13], and the majority of the adopted MPC based strategies can be considered as the velocity-prediction based methods, in which the solving algorithms, like DP [12] and estimation of distribution algorithm (EDA) [13], can search the optimal decision sequences within the receding prediction horizon. Intuitively, the effect of these solutions highly depends on the accuracy of velocity prediction. Even though some methods, such as back propagation neural network (BP-NN) [14], Markov chain (MC) [15] and deep neural network (DNN) [16], have been validated effective in precisely forecasting future velocity under certain conditions, improper prediction lengths and insufficient model training may deteriorate the application effect of the MPC strategies. In fact, MPC has been applied in vehicle control via reference-tracking for a long while [17, 18]. The step length of reference-tracking based MPC strategies can be less than $10 \mathrm{~ms}$ [17]. In the referencetracking based MPC, the complex control process in short horizons (e.g., the same or close to sampling time in engineering practice) can be achieved rapidly by efficient solvers with the target of simultaneously minimizing certain optimization targets and mitigating the difference between current state and reference [18]. Consequently, the referencetracking based MPC attains more promising capacity in realtime application than the velocity-prediction based long-term MPC. With more accurate reference in shorter receding horizon, the reference-tracking based MPC can search optimal solutions with higher efficiency [19]. Therefore, it is more appropriate to apply the reference-tracking based MPC in energy management problems in PHEVs. In current stage, to the best of authors' knowledge, the application of referencetracking based MPC has been widely applied in different fields, such as advanced vehicle dynamic control [20,21], and internal combustion engine (ICE) advance control [22, 23]. The homologous application in PHEV energy management, however, is quite rare.

By referring to the state-of-the-art discussion, an applicationoriented control strategy is proposed for a serial and parallel PHEV by employing the learning based MPC (LMPC) algorithm and microcosmic traffic flow analysis (MTFA). Rather than preferring the prediction-velocity based MPC in our former work [24], the reference-tracking based MPC manner is selected as the basic scheme. In [24], a synthesized velocity prediction method is introduced to facilitate the achievement of optimal control results, however the computation efficiency and application capability in practical environment is discounted. By contrast, the LMPC, with super capacity in reference-tracking based adaptive control, is applied to accomplish the energy flow management in the vehicle, raising efficient optimal solution in real time via minimizing the tracking gap and energy consumption. For providing the tracking reference for the LMPC based control, MTFA is applied to estimate future driving state on the basis of shared multi-source information. The simulation results validate the feasibility of the proposed LMPC algorithm and the MTFA based future driving state estimation technique. Two main contributions are added in the literature:

1) LMPC is applied in energy management within the powertrain of PHEV via reference tracking, and the robustness of LMPC in remedying uncertainties during state estimation is verified by the preferable energy management result.

2) MTFA is implemented to generate the reference for LMPC based strategy. Based on the diverse information shared through internet of vehicles (IoVs), MTFA offers accurate and detailed description on future driving state influenced by driving behaviors and surrounding environment.

The remainder of this paper is organized as follows. The studied PHEV and model construction are described in Section II. Section III elaborates the LMPC based strategy for energy management of PHEV. Section IV discusses the simulation results and comparatively validates the superior performance of the proposed control strategy. The main conclusions are drawn in Section V.

\section{THE STUdIED 4WD PHEV AND MODEL CONSTRUCTION}

The studied PHEV, as illustrated in Fig. 1, is with a serial and parallel configuration. The hybrid powertrain, including ICE, generator and motor, is fabricated in the front axle. The PHEV can operate in several modes through the cooperative operation among ICE, generator and motor. ICE can either supply tractive power through driving the generator in serial mode, or drive the vehicle directly with the motor in parallel mode. The switch between serial and parallel mode is attained by controlling the engagement of the clutch between ICE and motor. In addition, the battery can be charged or discharged in both serial and parallel modes governed by the control strategy. The detailed parameters are listed in Table I. 


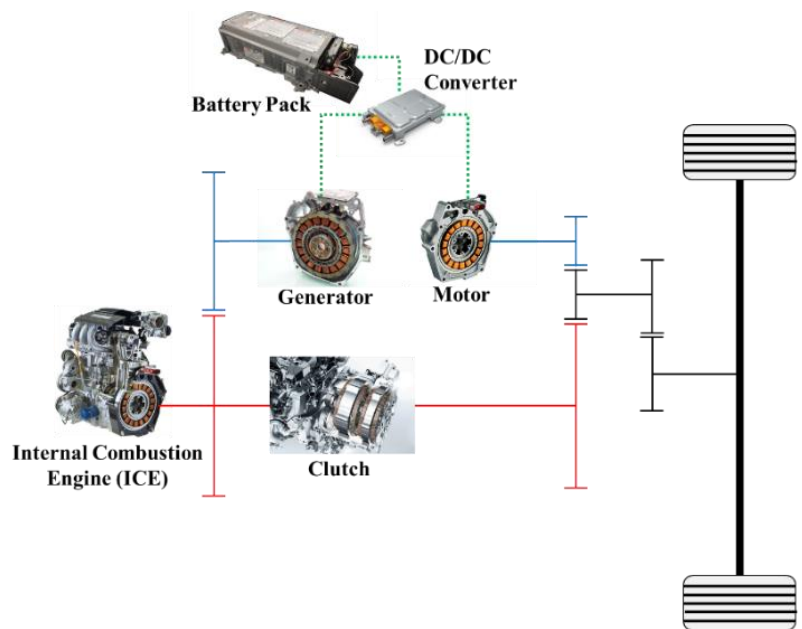

Fig. 1. The schematic of the serial and parallel PHEV configuration. TABLE I

COMPONENT PARAMETERS IN THE STUDIED PHEV

\begin{tabular}{|c|c|c|}
\hline \multirow{3}{*}{ ICE } & Displacement & $2.0[\mathrm{~L}]$ 16V DOHC \\
\hline & Maximum Power & 105[kW]@6200[rpm] \\
\hline & Maximum Torque & $\begin{array}{l}165[\mathrm{Nm}] @ 2500 \sim 6500 \\
{[\mathrm{rpm}]}\end{array}$ \\
\hline \multirow{2}{*}{$\begin{array}{l}\text { Motor and } \\
\text { Generator }\end{array}$} & Maximum Power & $124[\mathrm{~kW}]$ \\
\hline & Maximum Torque & $307[\mathrm{Nm}]$ \\
\hline \multirow{2}{*}{$\begin{array}{l}\text { Lithium-ion } \\
\text { Battery }\end{array}$} & Nominal Capacity & $15.7[\mathrm{kWh}]$ \\
\hline & Nominal Voltage & $300[\mathrm{~V}]$ \\
\hline \multirow{3}{*}{ Gear Ratio } & $\begin{array}{l}\text { Between ICE and final d } \\
\text { rive (gear } 1 \text { ) }\end{array}$ & $i_{g 1}=0.803$ \\
\hline & $\begin{array}{l}\text { Between motor and final } \\
\text { drive (gear 2) }\end{array}$ & $i_{g 2}=2.45$ \\
\hline & $\begin{array}{l}\text { Between ICE and genera } \\
\text { tor (gear } 3 \text { ) }\end{array}$ & $i_{g 3}=1.934$ \\
\hline
\end{tabular}

\section{a. Vehicle Dynamic Model}

Considering that the study is focused on only the longitudinal performance of PHEV, the lateral dynamics of PHEV is neglected. The vehicle longitude dynamics can be presented, as:

$$
\left\{\begin{array}{l}
a=\dot{v} \\
m a=\frac{1}{R_{w h}}\left(T_{r e q \_}-T_{b r k}\right)-\frac{1}{2} C_{D} A v^{2}-m g f \cos \alpha-m g \sin \alpha
\end{array}\right.
$$

where $v$ and $a$ denote the longitudinal velocity and acceleration, $m$ and $R_{w h}$ represent the vehicle mass and wheel radius, $\alpha$ and $f$ denote gradient and rolling resistance factor, $C_{D}$ and $A$ are the aerodynamic drag factor and frontal area, $g$ expresses the gravity acceleration, $T_{r e q_{-} d}$ and $T_{b r k}$ represent the tractive torque from the hybrid powertrains and mechanical braking torque. The tractive torque from the powertrain can be calculated as:

$$
T_{\text {req_d }}=T_{\text {fuel_path }}+T_{\text {ele }} \text { path }
$$

where $T_{\text {fuel_path }}$ and $T_{\text {ele_path }}$ denote the tractive torque provided by the fuel path and electric path. In different operation modes, the tractive torque from fuel path and electric path can be expressed as:

$$
\left\{\begin{array}{l}
T_{\text {fuel_path }}= \begin{cases}0 & E V / \text { Serial } \\
T_{\text {eng }} i_{g 1} \eta_{t_{-}{ }_{1}} i_{f d} \eta_{t_{-} f d} & \text { Parallel }\end{cases} \\
T_{\text {ele_path }}= \begin{cases}T_{e m} i_{g 2} i_{f d} \eta_{t_{-} g} \eta_{t_{-} f d} & E V / \text { Serial } \\
T_{e m} i_{g 2} \eta_{t_{-} 2} i_{f d} \eta_{t_{-} f d} & \text { Parallel }\end{cases}
\end{array}\right.
$$

where $T_{\text {eng }}$ and $T_{e m}$ respectively denote the engine torque and electric motor torque; $i_{f d}$ is the final drive ratio; $\eta_{t_{-} f d}, \eta_{t_{-} g 1}$ and $\eta_{t_{-} g}$ denote the mechanical transmission efficiency of final gear, gear 1 and gear 2, respectively. The mechanical transmission efficiencies of the mentioned three gears are respectively set to $0.91,0.92$, and 0.92 in the study.

\section{b. Engine Model}

In this study, the ICE's nonlinear performance is characterized by an efficiency map acquired from the benchmark test. The following equation shows the relationship between the engine net efficiency and torque, as:

$$
\eta_{\text {eng }}\left(T_{\text {eng }}, \omega_{\text {eng }}\right)=\frac{T_{\text {eng }} \omega_{\text {eng }}}{Q_{l h v} \dot{m}_{f}}
$$

where $\eta_{\text {eng }}$ is the engine efficiency, $\omega_{\text {eng }}$ means the rotating speed of engine, $Q_{l h v}$ represents the fuel lower heating value, and $\dot{m}_{f}$ expresses the fuel consumption rate. In serial mode, $\omega_{\text {eng }}$ is determined by interpolating the brake-special fuel consumption (BSFC) line according to specific torques. In parallel mode, $\omega_{\text {eng }}$ is calculated by:

$$
\omega_{\text {eng }}=\frac{\omega_{\text {wheel }}}{i_{f d 1} i_{g 1}}
$$

where $\omega_{\text {wheel }}$ denotes the rotating speed of wheel.

\section{c. Motor/Generator Model}

The motor and generator in the serial and parallel PHEV are all permanent magnet synchronous motors (PMSMs). PMSMs can operate either in tractive mode (functioned as motor) or generator mode. The dynamic characteristics of PMSMs are neglected due to the fast-transient responses and optimal control target. Likewise, the static models are also employed to describe the power performance of PMSMs, as:

$$
\left\{\begin{array}{l}
\eta_{\text {mot }}=\xi_{\text {mot }}\left(T_{\text {mot }}, \omega_{\text {mot }}\right) \\
\eta_{\text {gen }}=\xi_{\text {gen }}\left(T_{\text {gen }}, \omega_{\text {gen }}\right)
\end{array}\right.
$$

where $T_{m o t}$ and $T_{g e n}$ represent the torque of motor and generator; $\omega_{\text {mot }}$ and $\omega_{\text {gen }}$ mean the rotation speed of motor and generator; $\eta_{\text {mot }}$ and $\eta_{\text {gen }}$ denote the efficiency in tractive mode and generator mode; $\xi_{\text {mot }}$ and $\xi_{\text {gen }}$ describe the static map to calculate efficiency of motor and generator, respectively.

\section{d. Battery Model}

A simple equivalent circuit model is employed to characterize the electrical performance of battery. The 
temperature and ageing influence on battery performance is neglected for simplicity. The model consists of an open circuit voltage source, nonlinearly varying with state of charge (SOC), and an internal resistor connecting in series topology. The SOC can be calculated according to the simplified model, as:

$$
S \dot{O} C=-\frac{V_{o c}-\sqrt{V_{o c}-4 R_{\text {int }} P_{\text {batt }}}}{2 R_{\text {int }} Q_{\text {batt }}}
$$

where $S O C$ is the battery SOC, $V_{o c}$ is the open circuit voltage of battery, $R_{\text {int }}$ is the internal resistance of battery, $P_{\text {batt }}$ is the battery power, and $Q_{\text {batt }}$ is the battery capacity. The battery cells in the studied PHEV are lithium-iron phosphate battery, with a nominal capacity of 52.3 Ampere-hour (Ah). The nominal voltage of battery pack is $300 \mathrm{~V}$, with 90 cells connected in series topology. On the basis of the detailed powertrain modeling, the proposed LMPC based energy management will be addressed in the next section.

\section{The NOVEl CONTROL STRATEGY FOR PHEV}

\section{A. General Optimal Control Problem Formulation}

For attaining the optimal control by MPC through tracking reference, the nonlinear control problem can be generally defined as:

$$
U^{*}(k)=\operatorname{argmin} \sum_{i=0}^{h_{p}}\left\|y(k+i)-y^{*}(k)\right\|_{Q}^{2}+\|\Delta u(k+i)\|_{R}^{2}
$$

subject to:

$$
\left\{\begin{array}{l}
U(k) \triangleq\left[\Delta u^{T}(k), \Delta u^{T}(k+1), \ldots, \Delta u^{T}\left(k+h_{c}-1\right)\right]^{T} \\
\Delta u(k+i)=\Delta u\left(k+h_{c}-1\right), i=h_{c}, \ldots, h_{p} \\
Q=Q^{T}>0 \\
R=R^{T}>0 \\
\|x\|_{Q}^{2}=x^{T} Q x
\end{array}\right.
$$

where $u, x$ and $y$ are the control vector, state vector and control output; $y^{*}$ means the reference for tracking; $h_{c}$ and $h_{p}$ denote the control and prediction horizon; $A$ and $B$ are the system matrices; $Q$ and $R$ are the weight matrixes.

\section{B. Learning Based MPC}

The defined MPC based control problem in (8) can only achieve the optimal effect by precisely tracking the reference, which is supplied by the state prediction function, as:

$$
x(k+1)=f(x(k), u(k))
$$

From this point of view, the state prediction function is pivotal to capture system dynamics within prediction horizons and influence the control effect significantly. Furthermore, the online applications of MPC require that the state prediction function can describe future state accurately with less computation through simplifying the function, which, however, may lead to inferior control effects due to the raised prediction error. In addition, the powertrains in PHEVs are complex and nonlinear systems that are difficult to identify. As such, simple state prediction functions cannot accurately describe the comprehensive performance of PHEV powertrain in random conditions. Accordingly, the LMPC based control strategy is designed in this paper, and the accuracy of state prediction is significantly prompted after validation. The LMPC, compared with ordinary MPC, holds more ideal performance in complex control problems with the enhanced adaptability to complicate nonlinear systems [25]. Instead of using the fixed state prediction model, Gaussian process (GP) is integrated into the prediction model [26], allowing continuous update of the state prediction model to improve the estimating accuracy. On this account, the reference tracking precision is promoted, and the controlling performance is boosted [27]. By referring to the state prediction function, the learning process can be formulated, as:

$$
x(k+1)=f(x(k), u(k))+B_{d}(d(z(k)+w(k)))
$$

where $d$ is the leaning part of the prediction model that estimates the model error $d_{\text {true }}$ between the model and measurement, $z$ is the parameter vector related to the learning part, $w$ is the process noise, and $B_{d}$ is the weight matrix of the learning part. In the learning process in MPC, GP accounting for predicting values of leaning part is trained by the difference between the measured $x(k+1)$ and predictions by (10). The training data $t(k)$ can be expressed as [28]:

$$
t(k)=B_{d}^{+}(x(k+1)-f(x(k), u(k)))=d_{\text {true }}(z(k))+w(k)
$$

where $B_{d}^{+}$denotes the Morre-Penrose pseud-inverse matrix. The training data in (12) is the standard form of a regression task. Given the collected data $D(z)$, the GP model can stochastically estimate the regression results. With the updated state estimation by (11), MPC can be executed by some optimization methods, such as sequential quadratic programming (SQP) [29]. In GP, based on the parameter vector $z=\left[z_{1}^{T}, \cdots, z_{M}^{T}\right] \in \mathbb{R}^{M \times n_{z}}$ and the measurement vector $t=\left[t_{1}^{T}, \cdots, t_{M}^{T}\right] \in \mathbb{R}^{M \times n_{d}}$, the statistical model from the system can be expressed, as:

$$
t_{j}=g\left(z_{j}\right)+w_{j}
$$

where $w_{j}$ is the Gaussian noise with zero mean and diagonal variance, and $\Sigma_{w}=\operatorname{diag}\left(\left[\sigma_{1}^{2}, \cdots, \sigma_{n_{d}}^{2}\right]\right)$. The measurement is normally distributed with:

$$
[t]_{., a} \sim N\left(0, K_{Z Z}^{a}+\sigma_{a}^{2}\right)
$$

where $K_{Z Z}^{a}$ is the Gram matrix of the data points with the kernel function $k^{a}$. In this study, the selected specific kernel function is the squared exponential kernel function [30], as:

$$
k(z, \tilde{z})=\sigma_{f}^{2} \exp \left(-\frac{1}{2}(z-\tilde{z})^{T} L^{-1}(z-\tilde{z})\right)
$$

where $L \in \mathbb{R}^{n_{z} \times n_{z}}$ is the matrix with positive diagonal length. Given each output dimension $a \in\left(1, \cdots, n_{d}\right)$, the posterior distribution in dimension $a$ at a specific parameter group $z$ is the Gaussian function, of which the means and variance can be calculated, as: 


$$
\left\{\begin{array}{l}
\mu^{a}(z)=K_{z z}^{a}\left(K_{z z}^{a}+I \sigma_{a}^{2}\right)^{-1}[t]_{., a} \\
\Sigma^{a}(z)=K_{z z}^{a}-K_{z z}^{a}\left(K_{z z}^{a}+I \sigma_{a}^{2}\right)^{-1} K_{z z}^{a}
\end{array}\right.
$$

Now, the GP based regression analysis of the unknown function $g(z)$ can be yielded, as:

$$
d(z) \sim N\left(\mu^{d}(z), \Sigma^{a}(z)\right)
$$

where $\mu^{d}=\left[\mu_{1}^{d}, \cdots, \mu_{n_{d}}^{d}\right]$, and $\Sigma^{d}=\left[\Sigma_{1}^{d}, \cdots, \Sigma_{n_{d}}^{d}\right]$.

\section{Formulation of Optimal Energy Management in PHEV}

In LMPC based optimal energy management for PHEV, the control process to complete the optimal energy distribution is illustrated in Fig. 2, where the desired tractive torque $T_{r e q} d$ ordered from driver is regulated by the torque difference $T_{\Delta}$ generated from the receding optimization via LMPC. Then, the real required tractive torque $T_{r e q_{-} r}$, together with the torque distribution ratios among engine, motor and generator, is assigned to the powertrain to attain the energy distribution. The relationship between the desired tractive torque and real tractive torque as well as torque difference can be expressed as:

$$
T_{\text {req_r }}=T_{\text {req_d }}+T_{\Delta}
$$

According to the control process shown in Fig. 2, the control inputs of LMPC include the torque difference $T_{\Delta}$, the power distribution raito $\mu_{1}$ between engine and motor and the power distribution ratio $\mu_{2}$ between auxiliary power unit (APU) (consisting of ICE and generator) and motor. Here, the power distribution ratio $\mu_{1}$ and $\mu_{2}$ is respectively implemented in parallel and serial mode. The instant vehicle velocity and battery SOC are the state variables. Note that the studied PHEV will switch into charge sustaining stage from depleting stage when battery SOC is lower than 0.27.

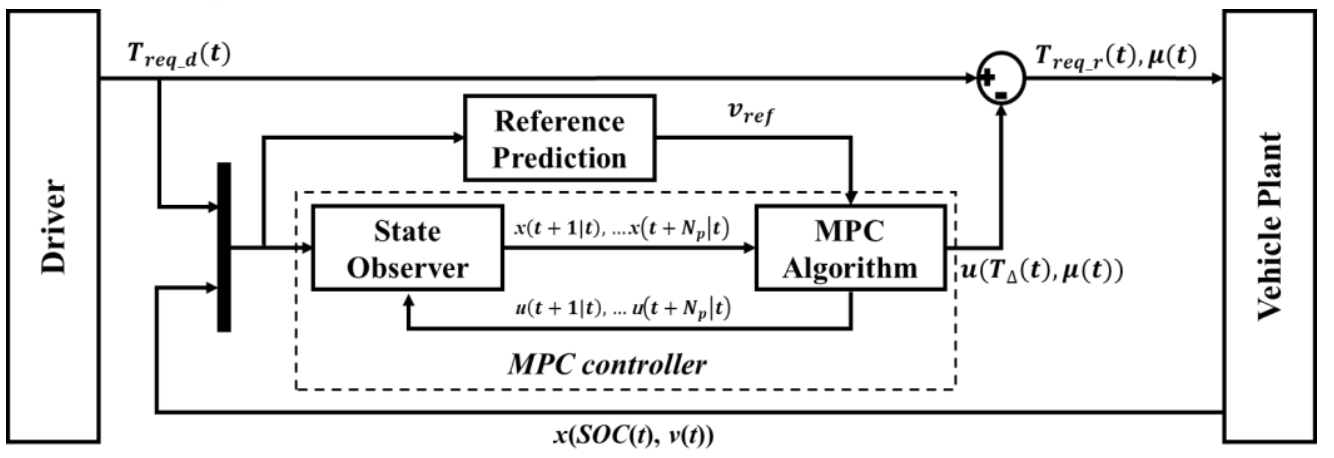

Fig. 2. Simplified architecture of the control strategy for PHEV.

The reference-tracking based LMPC in PHEV achieves the optimal control via efficient torque regulation and distribution. The torque regulation efficiently guides driving intention expressed in hybrid powertrain by referring to the estimated reference velocity. To avoid confliction with the original driving behaviors and driving comfort, the estimated reference velocity should be in line with the real driving in future. Therefore, the reference velocity estimation needs to be attained by the methods that can precisely describe the dynamics of future driving. The optimal control in PHEV for optimal energy management by reference-tracking based LMPC can be formulated as:

$$
U^{*}(k)=\arg \min \sum_{i=0}^{h_{p}} \varphi_{1} \dot{m}_{f}(t)+\varphi_{2}\left\|v(t)-v_{r e f}(t)\right\|^{2}+\varphi_{3} \frac{P_{b a t t}(t)}{Q_{l h v}}
$$

where $\varphi_{1}, \varphi_{2}$ and $\varphi_{3}$ are the weight ratio, and $v_{r e f}$ is the reference velocity. $P_{\text {batt }}$ can be calculated as:

$$
P_{b a t t}(t)=\left\{\begin{array}{cc}
\frac{\mu_{2} T_{\text {req_d }} n_{\text {wheel }}}{9550 \eta_{t_{-} f d} \eta_{t_{-} g} \eta_{e_{-} b m}} & \text { Serial Mode } \\
\frac{\mu_{1} T_{\text {req_d }} n_{w h e e l}}{9550 \eta_{t_{-} f d} \eta_{t_{-}{ }_{2}} \eta_{e_{-} b m}} & \text { Parallel Mode }
\end{array}\right.
$$

where $\eta_{e_{-} b m}$ and $\eta_{e_{-} g m}$ denote the electric transmission efficiency between motor and battery as well as generator and motor, respectively; and $\eta_{t_{-} g}$ is the mechanical transmission efficiency of gear 3. Moreover, the control should be subject to:

$$
\left\{\begin{array}{l}
S O C_{\min } \leq S O C(t) \leq S O C_{\max } \\
P_{\text {batt_min }} \leq P_{\text {batt }}(t) \leq P_{\text {batt_max }} \\
T_{\text {eng_min }} \leq T_{\text {eng }}(t) \leq T_{\text {eng_max }} \\
T_{\text {mot,gen_min }} \leq T_{\text {mot,gen }}(t) \leq T_{\text {mot,gen_max }} \\
w_{\text {eng_min }} \leq w_{\text {eng }}(t) \leq w_{\text {eng_max }} \\
w_{\text {mot,gen_min }} \leq w_{\text {mot,gen }}(t) \leq w_{\text {mot,gen_max }}
\end{array}\right.
$$

where the superscripts min and max denote the minimum and maximum value of variables. The internal state prediction models in LMPC need to be selected to describe key features of PHEV (e.g., vehicle dynamics and electricity consumption), as:

$$
\left\{\begin{array}{l}
\dot{v}(t)=\frac{1}{m}\left(T_{\text {req }} r(t)-\frac{1}{2} C_{D} A v^{2}(t)-m g f \cos \alpha(t)-m g \sin \alpha(t)\right)+B_{d_{-} d y n}\left(d\left(z_{d y n}(t)+w_{d y n}(t)\right)\right. \\
\operatorname{SOC}(t)=-\frac{V_{o c}(t)-\sqrt{V_{o c}(t)-4 R_{\mathrm{int}}(t) P_{\text {batt }}(t)}}{2 R_{\text {int }}(t) Q_{\text {batt }}}+B_{d_{-} \text {eny }}\left(d\left(z_{\text {eny }}(t)+w_{\text {eny }}(t)\right)\right.
\end{array}\right.
$$

where $B_{d_{-} d y n}$ and $B_{d_{-} e n y}$ denote the weight matrix, $w_{d y n}$ and $w_{\text {eny }}$ are the process noise, $z_{d y n}$ and $z_{\text {eny }}$ are the parameter vector, as:

$$
\left\{\begin{array}{l}
z_{\text {dyn }}=\left[v_{x}, a_{x}\right] \\
z_{\text {eny }}=\left[R_{\text {int }}, P_{\text {batt }}\right]
\end{array}\right.
$$


where $v_{x}$ and $a_{x}$ denote the velocity and acceleration in longitude direction, $R_{\mathrm{int}}$ and $P_{\text {batt }}$ represent the battery internal resistance and power, respectively. In the paper, the Gaussian model is trained based on the data acquired through the benchmark test. The data of velocity, acceleration in longitudinal direction, battery power, tractive torque, motor torque, ICE torque, and battery SOC are collected via dynamometer test based on the WLTC driving cycle. The battery internal resistance is measured on a battery cycler according to the test specifications. In the reference-tracking based LMPC for energy management of PHEV, the prediction horizon and the control step are set to $1 \mathrm{~s}$ and $0.1 \mathrm{~s}$, respectively; and the reference velocity is updated every $1 \mathrm{~s}$. The time parameters are chosen based on the real control process in onboard vehicle control units. By contrast, the prediction length of the velocity-prediction based MPC is set to $20 \mathrm{~s}$ to $50 \mathrm{~s}$ [31]. To guarantee the real-time application performance, the lengths of control step are enlarged to $1 \mathrm{~s}$ or longer. However, too larger control steps may not be implementable in real-time control. Even though the proposed LMPC based control strategy is developed based on the described PHEV configuration shown in Fig. 1, it can also be implemented to other PHEVs with different configurations, and only the powertrain parameters shown in Table I, the engine and motor efficiency maps, and the transmitting efficiencies in both fuel and electric path need to be updated. In addition, the inequality constraints described in (21) should also be adjusted accordingly.

\section{Reference Velocity Estimation by MTFA}

The required accurate estimation of reference velocity can be fulfilled only by integrally considering the influence from driving behaviors and surrounding environment. MTFA excels at describing the mentioned connection [32]. Even though microscopic traffic analysis has been widely investigated, integrating it into vehicle control, especially MPC based control, has not been fully investigated. MTFA proposed in this paper has considered much impact from environment and particular driver behaviors, making it rather suitable for MPC based control in PHEV. In this paper, the general expression of MTFA can be written as:

$$
m \ddot{x}_{n+1}(t+1)=\lambda \ddot{x}_{n+1}(t)\left[\frac{\dot{x}_{n}(t)-\dot{x}_{n+1}(t)}{\left(x_{n}(t)-x_{n+1}(t)\right)^{2}}\right](t-\Delta)
$$

where $\lambda$ is the sensitivity factor, $x$ denotes the vehicle position on the route, $m$ is the vehicle mass, $t$ is the time step, and $\Delta$ denotes the time lag. By solving (8), the velocity of target vehicle at time $t$ can be attained, as:

$$
v(t)=v_{f} \exp \left(\frac{\rho_{\text {jam }}}{\rho(t)}\right)
$$

where $v_{f}$ is the drag-free velocity, $\rho_{\text {jam }}$ is the jam density, and $\rho$ is the instant traffic flow density. The drag-free velocity can be physical characteristics of routes, which can be acquired through IoVs. The instant traffic flow density can be calculated as:

$$
\rho(t)=\frac{n}{\sum_{i=1}^{n} v_{i} \bar{h}(t)}
$$

where $v_{i}$ is the instant speed of certain vehicle on route segment, $n$ is the number of vehicles on route segment, and $\bar{h}$ is the average time headway. By substituting (10) into (9), the velocity of target vehicle can be written as:

$$
v(t)=v_{f} \exp \left(-\rho_{j a m} \bar{h}(t) \bar{v}_{r s}\right)
$$

where $\bar{v}_{r s}$ denotes the average speed of vehicles on the route segment that can be calculated as:

$$
\bar{v}_{r s}=\frac{\sum_{i=1}^{n} v_{i}}{n}
$$

Then, equation (27) can be extended by Taylor function [33], as:

$$
v(t)=v_{f}\left(1-\rho_{j a m} \bar{h}(t)\right) \bar{v}_{r s}+\frac{\left(\rho_{\text {jam }} \bar{h}(t) \bar{v}_{r s}\right)^{2}}{2 !}-\frac{\left(\rho_{\text {jam }} \bar{h}(t) \bar{v}_{r s}\right)^{3}}{3 !}
$$

According to (28), $\rho_{\text {jam }}$ and $\bar{h}$ are the key parameters affecting vehicle velocity in vehicle flow. $\rho_{\text {jam }}$ and $\bar{h}$, as a matter of fact, can be easily influenced by driving behaviors and driving environment, including traffic lights, crossroads, nearby buildings, etc. Therefore, the functions to describe the specific impact can be formulated as:

$$
\left\{\begin{array}{l}
\rho_{\text {jam }} \bar{h}(t)=\Gamma_{1}\left(v_{1} A^{2}+v_{2} A B+v_{3} B^{2}\right)+\Gamma_{2}\left(\zeta_{1} v+\zeta_{2} v a\right) \\
A=N_{t s}+N_{c} \\
B=N_{p u b}+N_{f}
\end{array}\right.
$$

where $N_{t s}, N_{c}, N_{p u b}$ and $N_{f}$ are the number of traffic lights, crossroads on the route segment and public buildings such as schools and hospitals and other buildings, respectively; $v_{1}, v_{2}$, $v_{3}, \zeta_{1}$ and $\zeta_{2}$ are system parameters; $\Gamma_{1}$ and $\Gamma_{2}$ are weight ratios. To obtain accurate values of the system parameters and weight ratios shown in (14), particle filters are employed to calculate the values based on the shared data from IoVs [34, 35]. According to (13) and (14), the reference velocity can be accurately estimated for the LMPC based energy management strategy. In IoVs, vehicles are equipped with enhanced communication techniques that can realize vehicle to vehicle $(\mathrm{V} 2 \mathrm{~V})$ communication and vehicle to infrastructure (V2I) communication [36]. The velocity of each vehicle on the route segment can be easily shared among multi-vehicles. Thus, $N_{t s}$, $N_{c}, N_{p u b}$ and $N_{f}$ can be statistically collected by infrastructures at road side and effectively disseminated among vehicles. Open data source like OpenStreetMap can also provide the environment information minutely [37], and we referred to the data therein for simulation.

\section{Simulation AND Evaluation}

To validate the performance of the proposed strategy, a series of simulations are performed [38]. In this study, the performance of MTFA in reference velocity estimation, the 
capability of LMPC in reference-tracking based optimal energy management in PHEV, and the energy-saving mechanism of LMPC are comprehensively evaluated through the comparison study. Note that the simulation is conducted on a workstation with an Intel i7-8700 processor and 16 Gigabytes memory.

\section{A. Performance Validation of MTFA in Reference Velocity Estimation}

The capability of MTFA is of the vital importance for LMPC based optimal energy management. The estimation accuracy of MTFA is comparatively studied among several methods, including participatory sensing data (PSD) based method [9], long-short term memory (LSTM) network [39] and support vector machine (SVM) [40]. Then, the stability and robustness of MTFA is verified among different road conditions. The city urban road (CR), highway (HW) and combined road (CW) conditions involving both $\mathrm{CR}$ and $\mathrm{HW}$ are selected for validation. The data for training models are derived from the collected data in real traffic test. To guarantee fair performance comparison and evaluation, all models are trained and evaluated by the same data set. Finally, the MPC controllers incorporating different velocity estimation methods are leveraged for energy management of the studied PHEV shown in Fig. 1, trying to
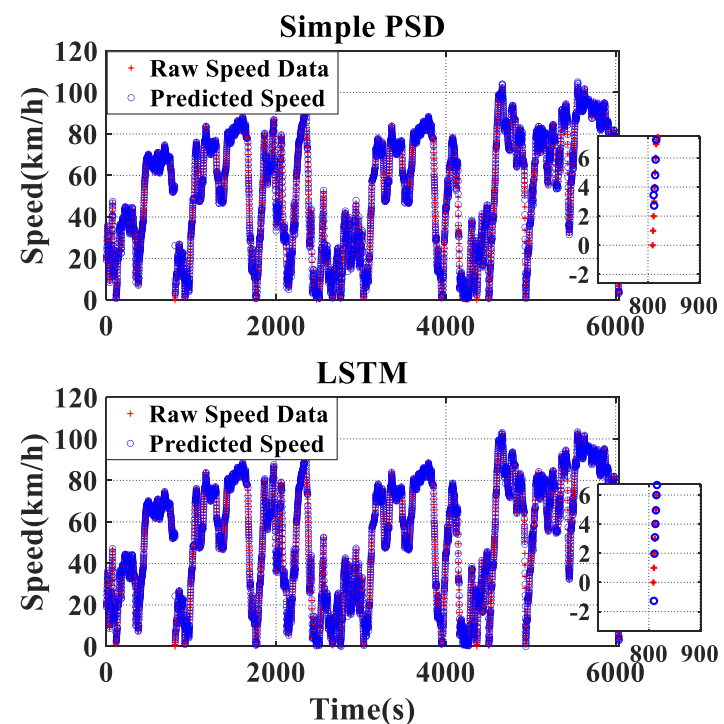

Fig. 3. Comparison in velocity estimation with different methods.

The numerical comparing results among different velocity estimation methods on the specified driving cycle are listed in Table II, where $R M S E$ and $M A E$ denote the root mean square error and mean absolute error respectively, as:

$$
\begin{aligned}
R M S E & =\sqrt{\frac{1}{m} \sum_{i=1}^{m}\left(y_{i}-\hat{y}_{i}\right)^{2}} \\
M A E & =\frac{1}{m} \sum_{i=1}^{m}\left|\left(y_{i}-\hat{y}_{i}\right)\right|
\end{aligned}
$$

where $y_{i}$ is the estimation value, $\hat{y}_{i}$ is the real value from the raw data. $T_{c a l}$ is the online processing time of CPU. The RMSE and MAE in Table II highlight that that LSTM and SVM methods perform slightly better than MTFA. However, the minor weakness in prediction accuracy cannot prevent MTFA evaluate the effect of the chosen velocity estimation methods by comparing the fuel economy of PHEV based on different controllers. During evaluation, the data for validating the MTFA and other baseline methods are extracted from the real traffic conditions according to the method described in [41].

Fig. 3 illustrates the estimated profiles by different methods together with the raw speed data. The estimation length of different methods is all set to $1 \mathrm{~s}$. To better present the behaviors of different methods, the zoomed-in figures sketching the estimated velocities around $800 \mathrm{~s}$ to $900 \mathrm{~s}$ are also provided. As can be found, most of the methods can achieve relatively high prediction accuracy, expect the simple PSD based method. The enlarged figures can partially facilitate the explanation of prediction accuracy discrepancy by different methods. The employed methods, include MTFA, LSTM and SVM, can capture more details than the simple PSD based method (as shown in the zoomed-in figures in Fig. 3). The promising abilities of LSTM and SVM in capturing future driving behaviors stem from the strong regression after fully understanding the inner connection between history data and future behaviors. While, the superior capability of MTFA to estimate future driving is incurred by the specific designed manners with the consideration of more environment influence.
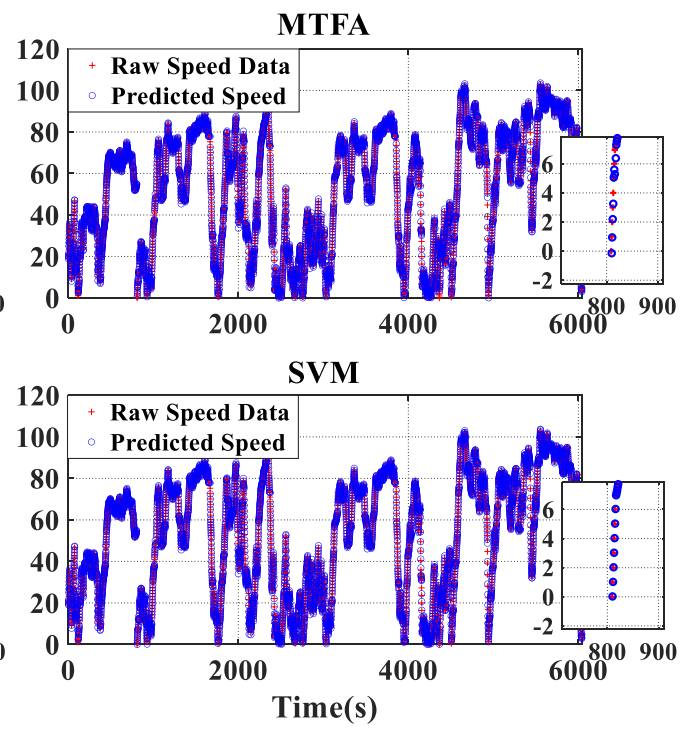

from being applied in real time due to much less online calculation time, compared with LSTM and SVM. Even though the simple PSD based method costs much less time (compared with other methods) because of its straightforward calculation manner, its prediction accuracy is obviously the worst among the chosen methods. The general comparison in Table II validates that MTFA is an ideal method to achieve the promising prediction accuracy in real time. To further investigate its stability and robustness in velocity estimation, the MTFA based method is tested to estimate velocities within 50 groups of data extracted from real traffic under different road conditions, as listed in Table III, where $R M S E_{a v a}, M A E_{a v a}$ and $T_{\text {cal_ava }}$ denote the average RMSE, MAE and CPU processing time; $R M S E_{\text {best }}, M A E_{\text {best }}$ and $T_{\text {cal_best }}$ denote the minimum RMSE, minimum MAE and shortest CPU processing time, respectively. 
The results in Table III show that the MTFA can estimate the vehicle velocity stably, and the average RSME, MAE and CPU processing time under different road conditions are quite close. The stable performance verifies the rational robustness of the MTFA method. The reason can be attributed to that the MTFA method can comprehensively consider the impact on future driving from different perspectives, and abundant information inclusion and carefully designed information integration manners all contribute to the performance improvement. Moreover, the reason why the performance of the MTFA method in CR is better than that in HW is that the amount of shared data in CR is more than that in HW, and certainly more data lead to better prediction precision.

$$
\text { TABLE II }
$$

Numerical Comparison in Prediction Accuracy

\begin{tabular}{llll}
\hline \hline Method & RMSE & MAE & $\boldsymbol{T}_{\text {cal }}(\mathbf{s})$ \\
\hline Simple PSD & 1.081 & 0.733 & 0.011 \\
\hline MTFA & 0.711 & 0.235 & 0.018 \\
\hline LSTM & 0.702 & 0.269 & 0.112 \\
\hline SVM & 0.692 & 0.223 & 0.107 \\
\hline \hline
\end{tabular}

TABLE III

Comparison of the MTFA Based Velocity Estimation

\begin{tabular}{|c|c|c|c|c|c|c|}
\hline Type & $R M S E_{\text {ava }}$ & $M A E_{\text {ava }}$ & $T_{\text {cal_ava }}$ & $R M S E_{\text {best }}$ & $M A E_{\text {best }}$ & $T_{c a l_{-} \min }$ \\
\hline$\overline{C R}$ & 0.731 & 0.249 & $0.021 \mathrm{~s}$ & 0.705 & 0.228 & $0.016 \mathrm{~s}$ \\
\hline HW & 0.793 & 0.262 & $0.026 \mathrm{~s}$ & 0.787 & 0.259 & $0.021 \mathrm{~s}$ \\
\hline Com & 0.766 & 0.254 & $0.023 \mathrm{~s}$ & 0.707 & 0.227 & $0.014 \mathrm{~s}$ \\
\hline
\end{tabular}

The comparison results in Table IV validate the performance of different velocity estimation methods in another perspective. The reference-tracking based MPC controllers employed in the studied PHEV all utilize the same solving algorithm (SQP), and the difference lies in only the integrated velocity estimation methods. By this manner, the capabilities of velocity estimation methods can be further assessed. The results in Table IV reveal that the fuel economy becomes better after the MPC controller integrates more accurate velocity estimation. The EFC in Table IV denotes the equivalent fuel consumption that includes the converted fuel consumption from electric energy. Considering the trade-off between the controlling effect and capability in real-time application, MTFA is most suitable for the MPC based strategy. Specifically, the difference in optimality between MPC with MTFA and MPC with SVM is only $0.1 \%$ when employing DP as the benchmark; however, the $T_{c a l}$ of MTFA is much less than that of SVM, highlighting its comprehensive feasibility in velocity prediction.

TABLE IV

Impact on Fuel Economy by Different Estimation Methods

\begin{tabular}{lll}
\hline \hline Method & EFC $(\mathbf{L} / \mathbf{1 0 0} \mathbf{k m})$ & Optimality $\mathbf{( \% )}$ \\
\hline MPC-Simple PSD & 2.664 & $95.07 \%$ \\
\hline MPC-MTFA & 2.634 & $96.17 \%$ \\
\hline MPC-LSTM & 2.633 & $96.21 \%$ \\
\hline MPC-SVM & 2.631 & $96.27 \%$ \\
\hline DP & 2.533 & 100 \\
\hline \hline
\end{tabular}

\section{B. Reference-Tracking Based Optimal Control in PHEV}

The raised LMPC can bring better performance in theory because of its better capability in inner state prediction after integrating the GP model. To more clearly demonstrate the remarkable performance of LMPC, a number of comparisons are conducted. In this part, MPC denotes the ordinary MPC application without the learning based state prediction function, and the solving manner is described minutely in [42]. Table V lists the fuel consumption by the ordinary MPC and LMPC with different velocity estimation methods. The MPC with SVM 10, SVM 20 and SVM 30 represents the velocity-prediction based ordinary MPC controller, in which the length of velocity prediction is $10 \mathrm{~s}, 20 \mathrm{~s}$ and $30 \mathrm{~s}$, respectively. Similarly, the LMPC with SVM 10, SVM 20 and SVM 30 expresses the velocity-prediction based LMPC controller, and the prediction length is the same as above. In addition, the MPC with MTFA and the LMPC with MTFA present the reference-tracking based ordinary MPC and LMPC controllers with MTFA as the velocity prediction algorithm, where the prediction length is set to $1 \mathrm{~s}$. Note that the control step of all the mentioned methods is $0.1 \mathrm{~s}$.

The numerical results listed in Table $\mathrm{V}$ underpin the conclusion that the LMPC controller outperforms the ordinary MPC controller. The equivalent fuel consumption by the LMPC controller is less than that based on the ordinary MPC controller. The accurate inner state prediction with GP models in LMPC supplies more chances to find optimal solutions and contributes to better fuel economy. Table $\mathrm{V}$ also lists the difference between the velocity-prediction based controller and referencetracking based controller. By referring to all ordinary MPC and LMPC controllers with different solving mechanisms, the reference-tracking based controller can lead to obvious superiority in real-time implementation. In the controllers with the reference-tracking based solving mechanism, the consumed calculation time in each step plus the time of reference estimation is shorter than the pre-given control step length, verifying its feasibility in instant application. For the velocityprediction based controller, however, only the CPU processing time in each step is much longer than the pre-defined control step length, thereby avoiding from instant real-time application. To apply the velocity-prediction based controllers in real time, the control steps should be preset with longer interval (such as $1 \mathrm{~s}$ ), which cannot satisfy the demand of engineering practice. Notably, the EFC by reference-tracking based controller is slightly more than that by the velocity-prediction based controllers. The reason of incurring minor difference is that the velocity-prediction based strategies consider impact of future driving on fuel economy within longer horizons, which is closer to the global optimal solutions. However, the maximum difference in EFCs is only $0.024 \mathrm{~L} / 100 \mathrm{~km}$, which can be neglected in instantaneous application.

To better demonstrate the difference in solving process between the ordinary MPC and LMPC, Table VI compares the solving performance of the ordinary MPC and LMPC. Both the ordinary MPC and LMPC execute the solution searching based on the reference-tracking principle, and exploit the proposed MTFA to estimate the reference. In Table VI, $\overline{S_{0}^{2}}$ denotes the average variance relating with state-constraint violations, and $\overline{\|e\|}$ means the difference between the predicted state after one step prediction and real state. The results show that the ordinary MPC without correction in inner state prediction leads to more constraint violation than that by LMP, which is mainly caused by the discretization error inherited from the inflexible state 
model. Smaller index $\overline{\| e} \|$ indicates that the GP model boosts the state prediction in LMPC. Thus, one can conclude that the GP model improves the accuracy of state prediction, thereby prompting the overall performance obviously.

TABLE V

Comparison of Fuel Economy by Ordinary MPC and LMPC with Different V elocity Estimation Methods

\begin{tabular}{llllll}
\hline \hline & Method & $\begin{array}{l}\text { EFC } \\
(\mathbf{L} / \mathbf{1 0 0 k m})\end{array}$ & $\begin{array}{l}\text { Optimality } \\
(\boldsymbol{\%})\end{array}$ & $\boldsymbol{T}_{\text {cal }}(\mathbf{s})$ & $\boldsymbol{T}_{\text {cal_step }}(\mathbf{s})$ \\
\hline \multirow{4}{*}{ MPC } & SVM 10 & 2.620 & 99.66 & 16622.10 & 0.330 \\
\cline { 2 - 6 } & SVM 20 & 2.617 & 99.77 & 19745.04 & 0.392 \\
\cline { 2 - 6 } & SVM 30 & 2.611 & 100 & 24983.52 & 0.496 \\
\cline { 2 - 6 } & MTFA & 2.634 & 99.13 & 3072.57 & 0.061 \\
\hline \multirow{3}{*}{ LMP } & SVM 10 & 2.589 & 99.67 & 16873.95 & 0.335 \\
\cline { 2 - 6 } C & SVM 20 & 2.587 & 99.75 & 19996.89 & 0.397 \\
\cline { 2 - 6 } & SVM 30 & 2.581 & 100 & 25336.11 & 0.503 \\
\cline { 2 - 6 } & MTFA & 2.603 & 99.16 & 3223.68 & 0.064 \\
\hline \hline
\end{tabular}

Solving Performance Comparison between MPC and LMPC

\begin{tabular}{lcl}
\hline \hline Method & $\overline{S_{0}^{2}}$ & $\overline{\|e\|}$ \\
\hline MPC-MTFA & 5.15 & 1.14 \\
\hline LMPC-MTFA & 1.06 & 0.08 \\
\hline \hline
\end{tabular}

\section{Energy-Saving Mechanism of LMPC based Strategy}

The results comparison in Table $\mathrm{V}$ validates the promising performance of LMPC, compared with the ordinary MPC. To further reveal the mechanism of the proposed LMPC based strategy in energy saving, the comparison study between the LMPC based method and a number of baseline control methods is performed, and the adopted baseline control strategies include the CD-CS scheme, ECMS, and DP, which can be described as follows.

1) The CD-CS scheme [4]. The CD-CS controller is a simple rule based strategy, in which the operation mode switches from CD to CS stage when battery SOC is less than 0.25 . In the CD stage, the battery supplies all the driving power except when the required tractive power is larger than an upper threshold; while in the CS stage, the ICE and battery work together to drive the vehicle.

2) ECMS [8]. In the ECMS based controller, the ICE and battery power combination that can contribute to the minimum instant equivalent fuel consumption is chosen as the current control policy. The equivalent factor in ECMS is tuned for given driving conditions by the specially designed optimization algorithm [43].

3) DP [6]. DP, as a well-known global optimization strategy, is chosen as the benchmark methods in most cases. In DP based controller, the control step and the numbers of state and control grid are respectively set to $0.1,700$ and 300 in this study.

Note that LMPC in following figures and tables denotes the raised LMPC based control strategy with the incorporation of MTFA, of which the main function is to provide reference velocity trajectories. The investigation in this part is addressed on a driving cycle extracted from real traffic data shown in [41]. Table VII lists the comparison on energy consumption by different methods. Apparently, the LMPC based strategy leads to less fuel consumption with the similar behaviors as DP. As can be found, the optimality of the LMPC based strategy can reach $96.21 \%$ of that by DP. The ECMS performs slightly worse than the LMPC based strategy, attaining $93.16 \%$ of that by DP. The CD-CS method, obviously, raises the worst performance among the applied methods, only reaching $80.23 \%$ that by DP. As shown in Fig. 4, the proposed LMPC based strategy behaves quite similarly with DP, and leads to close fuel consumption and battery power variation. Both DP and LMPC tend to coordinately distribute the power of ICE and battery, avoiding the operation of powertrain components in unfavorable area. The ECMS, partially incorporating environment information by tuning the equivalent factor [8], achieves better performance than the simple CD-CS method. The battery SOC by the simple rule based strategy drops fastest at the beginning of trip, and enables ICE to operate frequently after the vehicle untimely switches into the CS stage. By contrast, the LMPC based control strategy and DP can govern electric energy rationally consumed during the entire trip, and hinder frequent ICE operation engagement. The gap in energy consumption by different methods owns to the disperse integration level of environmental information. Given the full knowledge of driving cycle, DP can search the optimal solution to minimize the overall energy consumption. The LMPC, equipped with the prediction capabilities for future driving conditions in specified horizons, can attain quasi-optimal solutions and exhaustively reduce the energy consumption within the prediction trip. However, larger lengths of optimization horizons contribute to better control performance but with the price of computation intensity increase. The ECMS behaves worse than DP and LMPC, due to the limited ability in incorporating the time-varying influence incurred by driving environment in each step. The CD-CS scheme, even calibrated by expert knowledge and engineering practice, presents poor adaption to driving conditions and results in clumsy control effect.
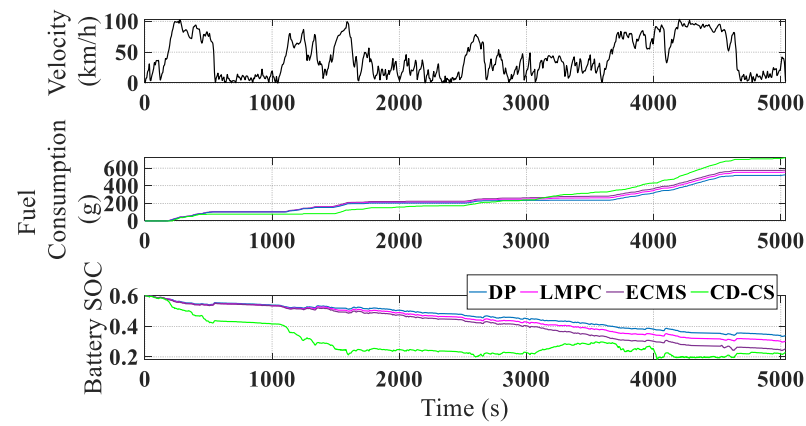

Fig. 4. Fuel consumption and battery SOC trajectories by different methods.

Fig. 5 present the powertrain operation modes by different methods on the given driving cycle. The proportions of operation time of each mode by DP and the LMPC based strategy are quite consistent. In contrast, the ECMS and CD-CS control strategy all switches into the CS stage before the trip ends, opposing to energy saving. The difference between DP and the novel LMPC in mode operation lies in that the LMPC algorithm prefers sporadic parallel mode, especially in sudden acceleration scenarios, due to the short prediction on future driving and the corresponding improper solving manner. To further compare the difference between the LMPC strategy and $\mathrm{DP}$, the powertrain component performance by the two control 
strategies are illustrated in Fig. 6. The powers of ICE, motor and generator by two methods are quite close, except some noticeable difference in acceleration stage, e.g. from $1401 \mathrm{~s}$ to 1681 s. DP, with the full knowledge of driving cycle, selects the serial assist mode to reach the minimum energy consumption in the whole trip. In contrast, LMPC is forced to run optimal solution searching within the narrow prediction horizon, enabling the direct ICE drive mode to avoid fast SOC decrease.

TABLE VII

Energy Consumption by Different Methods

\begin{tabular}{lllll}
\hline \hline Method & $\begin{array}{l}\text { Fuel } \\
\text { Consumpti } \\
\text { on (g) }\end{array}$ & $\begin{array}{l}\text { Electricity (k } \\
\text { Wh) }\end{array}$ & $\begin{array}{l}\text { EFC (L/10 } \\
\text { 0km) }\end{array}$ & $\begin{array}{l}\text { Optim } \\
\text { ality } \\
(\%)\end{array}$ \\
\hline CD-CS & 717.41 & 5.88 & 3.09 & 80.23 \\
\hline ECMS & 583.92 & 5.45 & 2.66 & 93.16 \\
\hline LMPC & 552.93 & 4.66 & 2.57 & 96.21 \\
\hline DP & 517.21 & 4.09 & 2.48 & 100 \\
\hline \hline
\end{tabular}

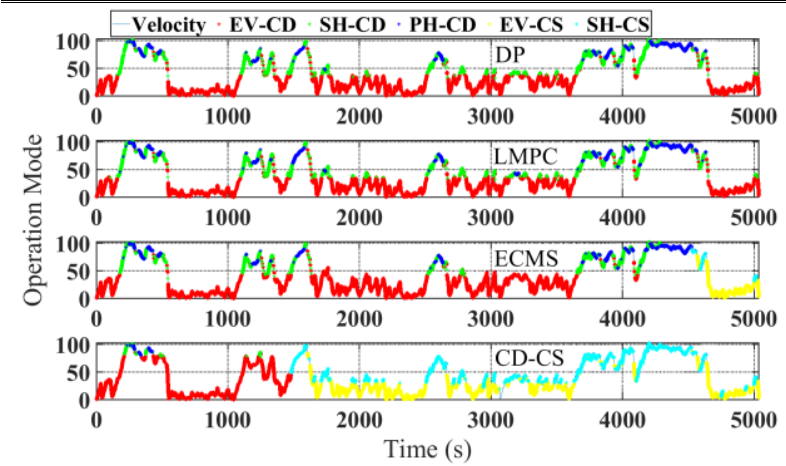

Fig. 5. Comparison of powertrain operation modes by different methods. EV$\mathrm{CD}$ and EV-CS respectively denotes pure electric mode in CD and CS stage; SH-CD and SH-CS expresses serial mode in CD and CS stage; PH-CD means the parallel mode in CD stage.
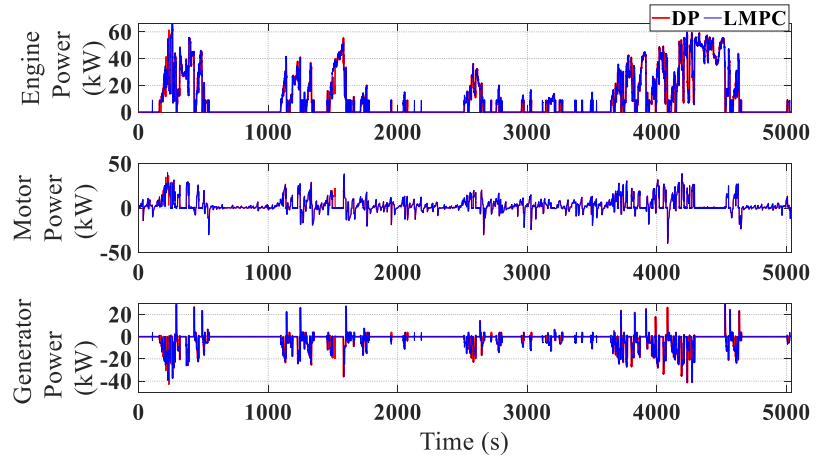

Fig. 6. Comparison of component performance by different methods.

Figs. 7 to 9 illustrate the powertrain performance by DP and LMPC based strategy in a sudden acceleration and deceleration segment. As shown in Fig. 7, DP and the LMPC algorithm result in almost the same fuel consumption rate except in the initial and middle acceleration stages. Although ICE is started more frequently by DP than by novel LMPC, DP enables more efficient operation of ICE than the novel LMPC algorithm, due to the benefit from pre-knowledge of the whole driving cycle. The LMPC strategy, limited by the finite predicted horizon, can only perform local optimal control within a short range, and fails to discover the minimum energy consumption in the whole trip. The difference in operation modes, as shown in Figs. 8 and 9 , supports the same conclusion. To be specific, DP prefers the serial assist mode, while the LMPC method urges to switch to the ICE direct drive mode. However, the LMPC strategy foresees upcoming continuous acceleration, thus prejudging that the high load driving condition is more suitable for ICE operation and battery SOC sustaining. Instead, DP, endowed with longer view of future deceleration, integrally exploits the serial assist mode, controlling the ICE to operate in higher efficiency region, and makes full use of regenerative braking energy to replenish electricity consumed in assist mode, as observed in Figs. 5 and 8. Note that the regenerative braking mode during deceleration is marked as the EV mode in both $\mathrm{CD}$ and CS stage for simplicity. To sum up, the proposed LMPC strategy can lead to preferable energy management in PHEV based on the strong local optimization and the receding horizon prediction capability.

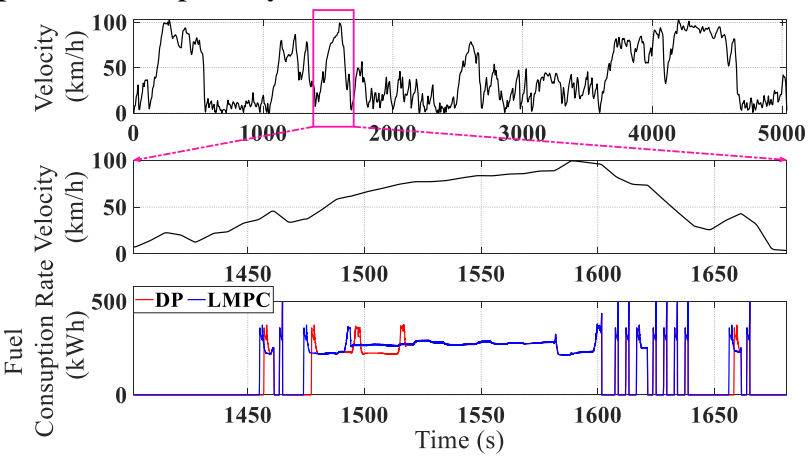

Fig. 7. The zoomed-in fuel consumption rate in sudden acceleration and deceleration conditions.

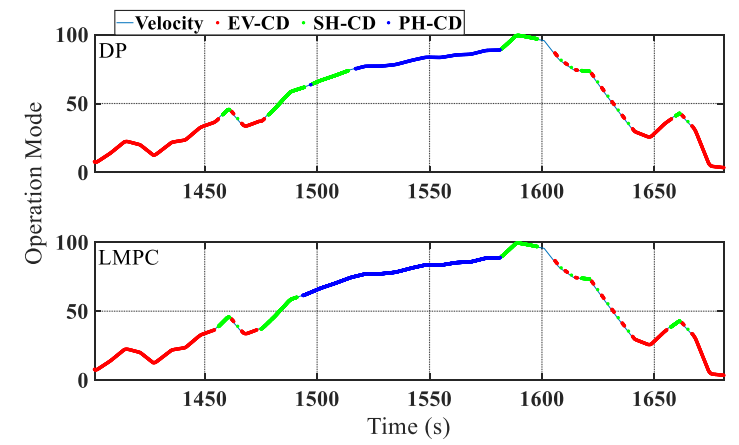

Fig. 8. The zoomed-in operation modes by DP and LMPC strategy in sudden a cceleration and deceleration conditions.
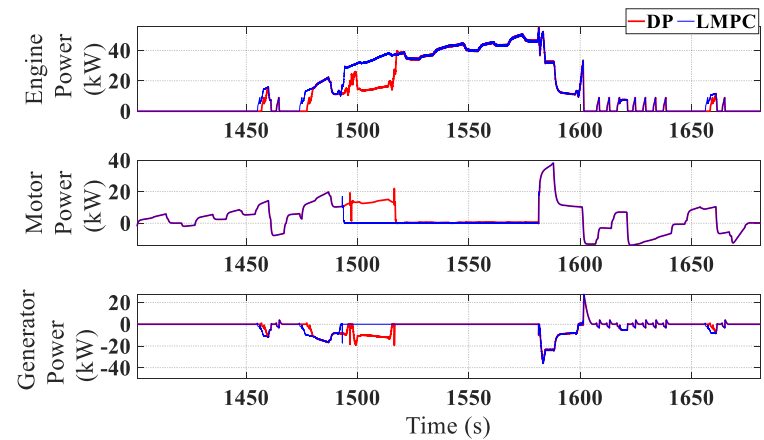

Fig. 9. The zoomed-in component performance by DP and LMPC based strate gy in sudden acceleration and deceleration conditions

\section{Validation in Other PHEVs with Different Configurations}

Even though the proposed LMPC strategy is developed for a serial-parallel PHEV, the feasibility of transplanting the raised method to other PHEVs with different configurations is also investigated in this study. The energy consumption by the LMPC with the MTFA based reference velocity estimation in 
different PHEVs is listed in Table VIII. In the comparison study, the selected serial PHEV, parallel PHEV, and 4WD PHEV are simulated and controlled by the designed algorithm. The detailed description on the configurations and component parameters can be found in our previous research [44-46]. In Table VIII, average EFC denotes the average equivalent fuel consumption of simulation on NEDC, UDDS, US06, HWFER, and JC 08 driving cycles. The average EFCs by the proposed LMPC based strategy is compared with those by the CD-CS scheme and DP. As can be found, the LMPC strategy presents stable performance in different PHEVs, showcasing its superior application capability and robustness. The maximum optimality resulted by the strategy reaches $95.79 \%$ of that by DP, and leads to significant promotion in all PHEVs, compared with the simple CD-CS algorithm.

TABLE VIII

Energy Consumption by Different Strategies in PHEVs with Different Configurations

\begin{tabular}{llll}
\hline \hline \multirow{2}{*}{ Vehicle Type } & Control Strategy & $\begin{array}{l}\text { Average EFC } \\
(\mathbf{L} / \mathbf{1 0 0 k m})\end{array}$ & $\begin{array}{c}\text { Optimality } \\
(\%)\end{array}$ \\
\hline \multirow{3}{*}{ Serial PHEV } & CD-CS & 18.02 & 81.63 \\
\cline { 2 - 4 } & LMPC-MTFA & 15.38 & 95.52 \\
\cline { 2 - 4 } & DP & 14.71 & - \\
\hline \multirow{3}{*}{ Parallel PHEV } & CD-CS & 3.54 & 82.29 \\
\cline { 2 - 4 } & LMPC-MTFA & 3.04 & 95.73 \\
\cline { 2 - 4 } & DP & 2.91 & - \\
\hline \multirow{2}{*}{ Serial-Parallel P } & CD-CS & 3.07 & 82.13 \\
\cline { 2 - 4 } HEV & LMPC-MTFA & 2.63 & 95.79 \\
\cline { 2 - 4 } & DP & 2.52 & - \\
\hline \multirow{3}{*}{ 4WD PHEV } & CD-CS & 4.51 & 87.42 \\
\cline { 2 - 4 } & LMPC-MTFA & 4.13 & 95.65 \\
\cline { 2 - 4 } & DP & 3.95 & - \\
\hline \hline
\end{tabular}

Through the comparison from different perspectives, the raised reference-tracking based LMPC controller shows obvious advantages in energy management of PHEV, and leads to preferable control effect with fast real-time implementation capacity. The specific reference-tracking manner fastens the computation speed, and moreover the leaning based state predictor and multi-source fused reference estimator improve the control effect significantly.

\section{CONCLUSION}

In this paper, a novel learning based control strategy is presented for PHEVs. The reference-tracking based MPC with leaning ability is preferred in the control strategy, possessing qualified capacity in real-time application. To promote the control effect, the Gaussian process model is integrated into the inner state predictor of MPC to mitigate the adverse effect on state prediction. The reference velocity in the leaning based MPC strategy is estimated by MTFA. The particular velocity estimation method can reasonably analyze the behaviors of future driving without much cost in calculation. The simulation evaluation demonstrates that the proposed leaning based control strategy is well-suited for the application in engineering practice with near-optimal effect. Compared with the rule based control strategy, the raised method can improve the fuel economy by as high as $15.98 \%$, and can achieve $96.21 \%$ fuel economy of that by DP. The algorithm is also validated effective in different types of PHEVs, manifesting its application capability and robustness.
As the future driving status tends to be easily affected by multiple factors from driving behaviors and environment, more effort will be devoted to velocity prediction in our future work. In addition, intelligent methods will be explored to incorporate more information from multi-sources for prediction precision promotion of future driving velocity.

\section{REFERENCES}

[1] Y. He et al., "Multiobjective Co-Optimization of Cooperative Adaptive Cruise Control and Energy Management Strategy for PHEVs," IEEE Transactions on Transportation Electrification, vol. 6, no. 1, pp. 346-355, Mar 2020.

[2] Y. Zhang et al., "Energy management strategy for plug-in hybrid electric vehicle integrated with vehicle-environment cooperation control," Energy, vol. 197, Apr 15 2020, Art. no. 117192.

[3] X. Zeng and J. Wang, "Optimizing the Energy Management Strategy for Plug-In Hybrid Electric Vehicles With Multiple Frequent Routes," IEEE Transactions on Control Systems Technology, vol. 27, no. 1, pp. 394-400, Jan 2019.

[4] X. Li and A. Simos, "Torque-Leveling Threshold-Changing Rule-Based Control for Parallel Hybrid Electric Vehicles," IEEE Transactions on Vehicular Technology, Article vol. 68, no. 7, pp. 6509-6523, Jul 2019.

[5] J. Li, Q. Zhou, H. Williams, and H. Xu, "Back-to-Back Competitive Learning Mechanism for Fuzzy Logic Based Supervisory Control System of Hybrid Electric Vehicles," IEEE Transactions on Industrial Electronics, vol. 67, no. 10, pp. 8900-8909, Oct. 2020.

[6] J. Liu, Y. Chen, W. Li, F. Shang, and J. Zhan, "Hybrid-Trip-Model-Based Energy Management of a PHEV With Computation-Optimized Dynamic Programming," IEEE Transactions on Vehicular Technology, vol. 67, no. 1, pp. 338-353, Jan 2018.

[7] S. Xie, S. Qi, and K. Lang, "A Data-Driven Power Management Strategy for Plug-In Hybrid Electric Vehicles Including Optimal Battery Depth of Discharging," IEEE Transactions on Industrial Informatics, vol. 16, no. 5, pp. 3387-3396, May 2020.

[8] Q. Guo, Z. Zhao, P. Shen, X. Zhan, and J. Li, "Adaptive optimal control based on driving style recognition for plug-in hybrid electric vehicle," Energy, vol. 186, Nov 1 2019, Art. no. 115824.

[9] Y. Zhang, L. Chu, Y. Ou, C. Guo, Y. Liu, and X. Tang, "A Cyber-Physical System-Based Velocity-Profile Prediction Method and Case Study of Application in Plug-In Hybrid Electric Vehicle," IEEE transactions on cybernetics, 2019-Aug-05 2019.

[10] C. Liu and Y. L. Murphey, "Optimal Power Management Based on QLearning and Neuro-Dynamic Programming for Plug-in Hybrid Electric Vehicles," IEEE Transactions on Neural Networks and Learning Systems, vol. 31, no. 6, pp. 1942-1954, Jun 2020.

[11] C. Song, H. Lee, K. Kim, and S. W. Cha, "A Power Management Strategy for Parallel PHEV Using Deep Q-Networks," in 2018 IEEE Vehicle Power and Propulsion Conference, 2018.

[12] S. Xie, X. Hu, Z. Xin, and L. Li, "Time-Efficient Stochastic Model Predictive Energy Management for a Plug-In Hybrid Electric Bus With an Adaptive Reference State-of-Charge Advisory," IEEE Transactions on Vehicular Technology, vol. 67, no. 7, pp. 5671-5682, Jul 2018.

[13] H. Lim and W. Su, "Hierarchical Energy Management for Power-Split Plug-In HEVs Using Distance-Based Optimized Speed and SOC Profiles," IEEE Transactions on Vehicular Technology, vol. 67, no. 10, pp. 9312-9323, Oct 2018.

[14] C. Sun, X. S. Hu, S. J. Moura, and F. C. Sun, "Velocity Predictors for Predictive Energy Management in Hybrid Electric Vehicles," IEEE Transactions on Control Systems Technology, Article vol. 23, no. 3, pp. 1197-1204, May 2015.

[15] C. Yang, S. You, W. Wang, L. Li, and C. Xiang, "A Stochastic Predictive Energy Management Strategy for Plug-in Hybrid Electric Vehicles Based on Fast Rolling Optimization," IEEE Transactions on Industrial Electronics, vol. 67, no. 11, pp. 9659-9670, Nov 2020.

[16] J. Q. Guo, H. W. He, J. K. Peng, and N. N. Zhou, "A novel MPC-based adaptive energy management strategy in plug-in hybrid electric vehicles," Energy, Article vol. 175, pp. 378-392, May 2019. 
[17] X. Wang, L. Li, and C. Yang, "Hierarchical Control of Dry Clutch for Engine-Start Process in a Parallel Hybrid Electric Vehicle," IEEE Transactions on Transportation Electrification, vol. 2, no. 2, pp. 231-243, Jun 2016.

[18] D. Zhao, R. Stobart, and B. Mason, "Real-Time Energy Management of the Electric Turbocharger Based on Explicit Model Predictive Control," IEEE Transactions on Industrial Electronics, vol. 67, no. 4, pp. 31263137, Apr 2020.

[19] Zhao, Dezong, Richard Stobart, and Byron Mason. "Real-time energy management of the electric turbocharger based on explicit model predictive control." IEEE Transactions on Industrial Electronics 67.4 (2019): 3126-3137.

[20] Zarkadis, Kinstantinos. Model predictive torque vectoring control with active trail-braking for electric vehicles. Diss. 2018.

[21] Siampis, Efstathios, Efstathios Velenis, and Stefano Longo. "Rear wheel torque vectoring model predictive control with velocity regulation for electric vehicles." Vehicle System Dynamics 53.11 (2015): 1555-1579.

[22] Moriyasu, Ryuta, et al. "Diesel engine air path control based on neural approximation of nonlinear mpc." Control Engineering Practice 91 (2019): 104114.

[23] Di Cairano, Stefano, et al. "Model predictive idle speed control: Design, analysis, and experimental evaluation." IEEE Transactions on Control Systems Technology 20.1 (2011): 84-97.

[24] Zhang, Yuanjian, et al. "A hierarchical energy management strategy based on model predictive control for plug-in hybrid electric vehicles." IEEE Access 7 (2019): 81612-81629.

[25] Manzano, José María, et al. "Robust learning-based MPC for nonlinear constrained systems." Automatica 117 (2020): 108948.

[26] C. E. Rasmussen, "Gaussian processes in machine learning," in Advanced Lectures on Machine Learning, vol. 3176, O. Bousquet, U. VonLuxburg, and G. Ratsch, Eds. Berlin: Springer-Verlag Berlin, 2004, pp. 63-71.

[27] Zhang, Kunwu, Qi Sun, and Yang Shi. "Trajectory Tracking Control of Autonomous Ground Vehicles Using Adaptive Learning MPC." IEEE Transactions on Neural Networks and Learning Systems (2021).

[28] C. K. I. Williams and C. E. Rasmussen, "Gaussian processes for regression," in Advances in Neural Information Processing Systems 8: Proceedings of the 1995 Conference, vol. 8, D. S. Touretzky, M. C. Mozer, and M. E. Hasselmo, Eds. Cambridge: M I T Press, 1996, pp. 514-520.

[29] M. Vajedi and N. L. Azad, "Ecological Adaptive Cruise Controller for Plug-In Hybrid Electric Vehicles Using Nonlinear Model Predictive Control," IEEE Transactions on Intelligent Transportation Systems, Article vol. 17, no. 1, pp. 113-122, Jan 2016.

[30] D. Duvenaud, H. Nickisch, and C. E. Rasmussen, "Additive Gaussian Processes," in neural information processing systems, 2011, pp. 226-234.

[31] S. B. Xie, X. S. Hu, Z. K. Xin, and J. Brighton, "Pontryagin's Minimum Principle based model predictive control of energy management for a plug-in hybrid electric bus," Applied Energy, Article vol. 236, pp. 893905, Feb 2019.

[32] Kessels, Femke, Kessels, and Rauscher. Traffic flow modelling. Springer International Publishing, 2019

[33] Zhang, Zhen, et al. "Continuous monitoring of residual torque of loose bolt in a bolted joint." Procedia Engineering 188 (2017): 278-285.

[34] D. Yuan, X. Lu, D. Li, Y. Liang, and X. Zhang, "Particle filter re-detection for visual tracking via correlation filters," Multimedia Tools and Applications, vol. 78, no. 11, pp. 14277-14301, Jun 2019.

[35] W. Li, Z. Wang, Q. Liu, and L. Guo, "An information aware eventtriggered scheme for particle filter based remote state estimation," Automatica, vol. 103, pp. 151-158, May 2019.

[36] F. Lyu et al., "Intelligent Context-Aware Communication Paradigm Design for IoVs Based on Data Analytics," IEEE Network, vol. 32, no. 6, pp. 74-82, Nov-Dec 2018.

[37] M. Haklay and P. Weber, "OpenStreetMap: User-Generated Street Maps," IEEE Pervasive Computing, vol. 7, no. 4, pp. 12-18, Oct-Dec 2008.

[38] X. Ding et al., "Analytical and Experimental Evaluation of SiC-Inverter Nonlinearities for Traction Drives Used in Electric Vehicles," IEEE Transactions on Vehicular Technology, vol. 67, no. 1, pp. 146-159, Jan 2018.

[39] Memarzadeh, Gholamreza, and Farshid Keynia. "A new short-term wind speed forecasting method based on fine-tuned LSTM neural network and optimal input sets." Energy Conversion and Management 213 (2020): 112824
[40] Kouziokas, Georgios N. "SVM kernel based on particle swarm optimized vector and Bayesian optimized SVM in atmospheric particulate matter forecasting." Applied Soft Computing 93 (2020): 106410.

[41] Hongwen, He, et al. "Real-time global driving cycle construction and the application to economy driving pro system in plug-in hybrid electric vehicles." Energy 152 (2018): 95-107.

[42] Grüne, Lars, and Jürgen Pannek. "Nonlinear model predictive control." Nonlinear Model Predictive Control. Springer, Cham, 2017. 45-69.

[43] Mirjalili, Seyedali, and Andrew Lewis. "The whale optimization algorithm." Advances in engineering software 95 (2016): 51-67.

[44] Zhang, Yuanjian, et al. Optimal Control Strategy for the Next Generation Range Extended Electric Bus. No. 2020-01-0844. SAE Technical Paper, 2020

[45] Zhang, Yuanjian, et al. "Energy management strategy for plug-in hybrid electric vehicle integrated with vehicle-environment cooperation control." Energy 197 (2020): 117192.

[46] Zhang, Yuanjian, et al. "A novel optimal power management strategy for plug-in hybrid electric vehicle with improved adaptability to traffic conditions." Journal of Power Sources 489 (2021): 229512. 